\title{
Carbon Sorbent of Destructive Type Based on Wood Biochar for Removal of Oil Pollution
}

Khokhlov Andrii Viktorovich and Khokhlova Lyudmila losiphovna

Institute for Sorption and Problems of Endoecology, NAS of Ukraine, Ukraine

\begin{abstract}
The peculiarities of obtaining biochar from coniferous wood species with a hydrophobic surface for immobilization of oil-oxidizing microorganisms in the creation of an oil-sorbent of destructive type are studied. The influence of different conditions of heat treatment of wood raw materials on the sorption properties of carbon material in relation to oil and microorganisms is studied. It was found that such a bio-coal has an oleophilic surface and has significant sorption properties with respect to oil. The carbon material is biocompatible.
\end{abstract}

Keywords: Carbon sorbent; Oleophilic matrix; Pyrolysis of wood; Hydrophobicity; Oil-oxidizing microorganisms

\section{Introduction}

Existing technologies for eliminating the effects of oil pollution using various sorbing materials, both natural and synthetic, are limited to the removal of petroleum products. Conventional sorbents create great difficulties in their application. Oil-absorbing sorbents at the collection of oil and oil products form multi-kilogram oil conglomerates that are difficult to collect from the surface of water or soil. In addition, the negative effect also arises from the sorbents themselves, the basis of which sometimes introduces additional pollution into the environment. A promising direction is the development of technologies using bioactive sorbents possessing physical and chemical and sorption activity with respect to oil and petroleum products, as well as the ability to decompose oil products by biodegradation. Bioactive sorbents based on absorbent material with oil-oxidizing microorganisms immobilized on its surface are able to localize oil pollution and neutralize it in a localized state. The destruction of oil can be carried out until the final stage, when only ecologically inert oil decomposition products remain. The rest of the biosorbent is the starting material base. The use of bioactive sorbents will quickly and effectively localize spills of oil and oil products and, in addition, ensure the complete decomposition of the latter.

The development of bioactive sorption materials based on raw materials of natural origin, bioactivated by a natural complex of oiloxidizing microorganisms with a wide spectrum of action is actual. The pore space of the biochar serves as a refuge for microorganisms. It is assumed that this is due to the influence of biochar on the growth of microbial biomass, as well as the growth of its activity [1-3]. Ecologically safe materials of destructive type will ensure complete localization of oil pollution and its decomposition in a localized state. Obtaining biologically active environmentally friendly oil sorbents based on raw materials of natural origin requires the study of adsorption, the physicochemical patterns of interaction between the sorption matrix and immobilized microorganisms-oil destructors.

\section{Materials and Methods}

To obtain a sorption matrix for immobilization of oil-oxidizing microorganisms, a bio-carbon material based on pyrolyzate of wood raw materials was used. The following were used as research objects: pyrolysates of various types of wood obtained under certain pyrolysis regimes; crude oil of different chemical composition; oxidized, weathered oil of oil sludge; oil products of various types; culture of oiloxidizing microorganisms isolated from oil contaminated objects and adapted over a wide range of temperatures for various types of oil and oil products. In practical use, it is necessary to take into account the requirements for oil sorbents, namely: the sorbent must:

- be hydrophobic and absorb a large amount of oil per unit of mass;

- adsorb the minimum amount of water;

- possess high sorption capacity and retaining capacity;

- be non-toxic.

Investigations of physicochemical and structural-sorption properties of the carrier matrix were carried out by the method of X-ray Phase Analysis and IR spectroscopy. The specific surface area of the carrier and biosorbent was studied by the express method of thermal desorption of argon. The volume of sorption of the pores of the carrier matrix and biosorbent was determined by the express method for sorbing benzene vapor. Statistical exchange capacity - sample treatment with $\mathrm{NaOH}$ solution. The sorption capacity relative to the oil was determined by a weighting method based on the difference in weight of the oil-saturated and original sample. Criteria for the effectiveness of the biosorbent were estimated by the amount of sorbed oil and residual after destruction by the method of extraction of absorbed oil from the surface, followed by photocolorimetric measurement and calculations of the absorbed total liquid, oil and water [4,5]. The chemical composition of oil and petroleum products before and after destruction was determined by chromatographic method. The development of the microbial process, the number of microorganisms studied by the method of limiting dilutions and determination of the optical density of the liquid.

\section{Results}

Carbon sorbent based on pyrolyzate from cellulose-containing plant raw materials (wood) among powdered sorbents of natural origin has

*Corresponding author: Khokhlova Lyudmila losiphovna, Institute for Sorption and Problems of Endoecology, NAS of Ukraine, Ukraine, Tel: +380442396444 ; E-mail: khokhlova.lyudmila@gmail.com

Received July 19, 2018; Accepted July 26, 2018; Published July 30, 2018

Citation: Viktorovich KA, losiphovna KL (2018) Carbon Sorbent of Destructive Type Based on Wood Biochar for Removal of Oil Pollution. J Environ Anal Toxicol 8: 576. doi: 10.4172/2161-0525.1000576

Copyright: ( 2018 Viktorovich KA, et al. This is an open-access article distributed under the terms of the Creative Commons Attribution License, which permits unrestricted use, distribution, and reproduction in any medium, provided the original author and source are credited. 
Citation: Viktorovich KA, losiphovna KL (2018) Carbon Sorbent of Destructive Type Based on Wood Biochar for Removal of Oil Pollution. J Environ Anal Toxicol 8: 576. doi: 10.4172/2161-0525.1000576

Page 2 of 3

a satisfactory absorption capacity for oil and petroleum products of different content and is biocompatible. Pyrolysis of cellulose-containing plant raw materials in strictly functional mode allows obtaining biocoal with certain properties with respect to oil and petroleum products. The final product of complete pyrolysis of raw materials is almost pure carbon, containing minor admixtures of potassium, sodium, magnesium calcium and iron oxides. Pyrolysis is based on a free-radical reaction of thermal destruction of hemicelluloses, cellulose and lignin occurring in the temperature range 473.15-523.15, 513.15-623.15 and 523.15-673.15 K. Heat treatment at a temperature below $523.15 \mathrm{~K}$ does not allow obtaining a stable porous structure. Heat treatment at a temperature of more than 673.15 leads to some reduction in the total volume and a change in the proportion of meso- and macropores from the total pore volume. Structural and chemical transformations in the process of pyrolysis of primary raw materials containing cellulose determine the properties of the resulting carbonaceous material. Thermal oxidation of a primary material in an oxygen-free atmosphere produces amorphous carbon. If pyrolysis time is not enough, then there are X-ray two broad gallo, typical for cellulose with max. 5.52 and 3.97 A. An increase in the pyrolysis time and a temperature of more than $673.15 \mathrm{~K}$ leads to the destruction of the structure of amorphous coal, the formation of ash, the crystallization of inorganic compounds ( 4.5 $\mathrm{A}^{\circ}$ ), and the deterioration of the sorption properties of the material. Previously, amorphous modification of carbon in coal from such a raw material was proved $[6,7]$.

Optimal conditions for the pyrolysis of raw materials were established in the laboratory. The optimum pyrolysis temperature is $573.15-623.15 \mathrm{~K}$, and the duration of the pyrolysis process is $25-30$ minutes, which allows obtaining high-quality carbon material for oil absorption and immobilization of oil-oxidizing microorganisms. When thermal oxidation of coniferous species of wood in a functional mode, wood bio-carbon is formed, where the carbon skeleton is saturated with resin. Unsaturated carbon atoms are bound to molecules of resinous substances. The surface of the carbon sorbent becomes hydrophobic and exhibits sorption properties with respect to oils. Absorption capacity of the sorbent has an extreme dependence on time and pyrolysis temperature and reaches values $>8 \mathrm{~g} / \mathrm{g}$.

The nature of the surface of the carbon sorbent affects both the sorption properties and the possibility and character of the immobilization of the microorganism-oil destructors on the surface of the sorption material. The reactive groups $\left(-\mathrm{COOH},-\mathrm{NH}^{2-},-\mathrm{N}_{2}+\right.$ and $-\mathrm{NCO}^{-}$) formed on the surface of the coal during pyrolysis determine the ability to retain biomolecules on the surface.

IR spectroscopic studies of the carbon sorbent before and after bioactivation showed a decrease in the intensity of the band (1200$1300 \mathrm{~cm}^{-1}$ ), responsible for the fluctuations of the phenolic groups and the intensity of the band $\left(1700-1750 \mathrm{~cm}^{-1}\right)$, characteristic of carboxyl groups. There is a surface interaction of the sorption matrix with the oil-destroying biocomplex. This fact confirms the possibility of using a carbon material to produce a bioactive sorption destructive type.

The study of the structural characteristics of the initial carbon matrix and after bioactivation showed changes in the specific surface area and the amount of oxygen-containing functional groups of the protogen. Absorbed biomolecules are retained on the surface of the carrier due to van der Waals forces, hydrogen bonds, and hydrophobic interactions.

The culture of oil-oxidizing microorganisms for bioactivation the carbon sorbent, isolated from natural complexes, included a wide range of microorganisms-destructors of aerobic and microaerophilic type, stable under unfavorable environmental conditions (temperature, high pollutant concentrations, etc.). Under natural conditions, the formation and accumulation of positive properties of microorganisms takes place. To obtain a microbial complex of natural origin, investigations were carried out on the isolation, selection and selection of oil-oxidizing microbiota from samples of oil-contaminated soils, sewage, oil trap for treatment plants, mud sludge of oil sludge accumulators, sediments from settling tanks of waste drilling fluids from industrial sites of oil fields. In order to increase the biodestructive efficiency of the whole group of oil-oxidizing microorganisms, the microbial mixture was adapted to various types of oil and petroleum products and to low temperatures. Cumulative cultures of microorganisms were grown on ordinary elective media at temperatures of 303.15 to $308.15 \mathrm{~K}$ in the presence of a hydrocarbon of oil, taking into account the optimum development of their development. For bioactivation of the carbon carrier, the most active component of the native culture was selected.

It is established that when the microbial population is adapted, physiological changes have a specific, purposeful character and capture the bulk of the microbial population. These changes occur within the limits of the hereditary rate of reactions without changing the basic hereditary properties. The longer cells are subjected to negative influence, the more strengthened and fixed in them the corresponding changes. This fact is used in the preparation of a broad-spectrum microbial concentrate. The nutrient medium in the microbial suspension provides optimal oxidation-reduction conditions, the necessary $\mathrm{pH}$, osmotic pressure, ion equilibrium. Nutrients are chemical compounds that are incorporated into the internal cellular metabolism. These are mineral salts, microelements, organic matter (oil), which are digested by microorganisms.

The rate of bacterial action depends on the total surface area of hydrocarbons that are degraded by microorganisms. The greatest activity of the action is manifested when hydrocarbons are dispersed and the greatest plane of the contact surface per unit mass is provided. Dispersing or emulsifying hydrocarbons contributes to the active development of biocenosis. Immobilization of microorganisms on the surface of the sorbent provides elective conditions for biodegradation of oil in the sorbed state. The use of microorganism cultures from the environment has several advantages: first, the original natural population is well adapted to environmental conditions; secondly, high stability and synergy can ensure a more complete use of the target substance as the sole source of nutrition; thirdly, the return of the microorganism to the initial environment gives an elective advantage. Studies have shown that the immobilization of the surface of the carbon sorption matrix should be only partial, so as not to reduce the sorption capacity of the material relative to oil.

It was found that with the number of microorganism cells 120-200 $\times 10^{4} \mathrm{cells} / \mathrm{g}$ on the surface, the maximum sorption of biosorbent is 8 $\mathrm{g} / \mathrm{g}$; when the number of cells is $200-300 \times 10^{6}$ cells $/ \mathrm{g}$, the maximum sorption is $6 \mathrm{~g} / \mathrm{g}$; and with the number of cells $-200-300 \times 10^{9} \mathrm{cells} / \mathrm{g}$, the sorption of the biosorbent decreases to $-4 \mathrm{~g} / \mathrm{g}$. Experimental data show that an increase in the concentration of cells on the surface of a carbon sorbent carrier affects the biodegradation of sorbed oil only in the initial period, but significantly reduces the sorption capacity of the carbon sorption matrix.

Determination of the degree of destruction of petroleum hydrocarbons was carried out by direct measurement of quantitative changes and the disappearance of hydrocarbons. An adequate indicator of bacterial growth during biodegradation can be the determination of 
microbial mass in water. The change in the state of the environment due to the vital activity of bacteria causes bacterial turbidity. Comparative determination of residual oil (extraction and photo-colorimetric measurements) and bacterial turbidity (measurement of turbidity or weight of dry biomass after centrifugation) allow us to characterize the development of the microbial substance and the degree of biodegradation of oil. Experimental data showed the destruction of $99 \%$ of the oil localized on the biosorbent. The final products of oil destruction are carbon dioxide and water. The residual product of the carbon biosorbent is a safe wood ash. The complex approach to the solution of the problem of liquidation of oil pollution - sorption+microbial destruction by the use of carbon sorbent of destructive type based on wood biochar will increase the ecological efficiency and safety of the technology.

\section{Conclusions}

The carried-out researches have shown that the carbon matrix based on wood biochar is perspective for obtaining sorbents of oil of destructive type. It has been established that the sorption properties of the carbon matrix depend on the type of wood raw material, the temperature and time of pyrolysis, the composition of the feedstock and pyrolyzate. The best sorption characteristics for oil are carbon material based on pyrolyzate of coniferous species of wood. The main factor of the sorption capacity of the matrix sorbent is the interaction of its hydrophobic surface with the oil. The carbon sorbent absorbs oil and oil products and exhibits biocompatibility with oil-oxidizing microorganisms. Water-oil-sorbent is a heterogeneous system. In this case, there is a practical use of hydrophobic interaction in heterogeneous systems. The hydrophobic interaction in such a system depends on the nature of the sorbent particles and the degree of their hydrophobicity. Pyrolyzate of pine is characterized by hydrophobicity of $98 \%$. The behavior and properties of the system where hydrophobic particles are present are determined completely hydrophobic interaction between hydrophobic particles of a sorption material and a hydrocarbon substance.

The carried-out researches of structural-sorption properties of biosorbents of carbon materials testify to the possibility of using such a material as a matrix for the immobilization of oil-destroying microorganisms in the production of biosorbents. For bioactivation of the carbon carrier, the most active component of the native culture was selected. Microbial colonies were obtained from the inoculum by the method of enrichment and selection on a nutrient medium of a certain composition. Enrichment was carried out in a wide range of temperatures on various types of oil and oil waste. Adaptation of the obtained microbial suspension to aerobic and microarophilic conditions, to lowered temperatures was conducted. The cultivation of microorganisms in the pores on the surface of the carrier mimics their natural existence. The use of inoculum from the environment causes their selective advantage and a high degree of destruction of oil localized on the sorbent.

The rate of bacterial action depends on the total surface area of hydrocarbons that are degraded by microorganisms. The greatest activity of the action is manifested when hydrocarbons are dispersed, when the greatest plane of the contact surface per unit of mass is provided, because the bacteria exist and feed mainly in the aquatic environment and act only upon contact of water and hydrocarbons. Immobilization of microorganisms-destructors on the surface of a carbon oil absorbing sorbent was carried out by treating the sorbent with a microbial suspension on an agarized basis, which contained a certain amount of adapted seed culture of microorganisms and nutrients. Thanks to adsorption on the surface of hydrophobic coal particles of organic (oil) and mineral nutrients, centers of microbial action are created. Growth of microorganisms on the sorbent is a heterophase cultivation of microorganisms.

For the cultivation of the bacterial culture the following conditions were provided:

- viable seed material (microbial concentrate);

- energy and carbon sources (oil and oil products);

- all additional nutrients necessary for biomass growth;

- the appropriate physico-chemical conditions in the culture medium.

\section{References}

1. Jeffery S, Abalos D, Spokas KA (2015) Biochar for Environmental Management: Science, Technology and Implementation. 2nd edn. Lehmann, pp: 301-326.

2. Keith A (2011) Interactive priming of biochar and labile organic matter mineralization in a smectite-rich soil. Environmental Science and Technology 45: 9611-9618.

3. Lehmann J (2011) Biochar effects on soil biota-a review. Soil Biology and Biochemistry 43: 1812-1836.

4. Gridin OM (1996) Oil filling and saving sorbents 5: 10-13.

5. Mochalova OI (1992) Physical and chemical methods for dealing with oil spills Oilman 12: 24-27.

6. Shvets DI, Khokhlova LI, Kravchenko OV, Openko NM, Khokhlov AV (2002) Physical and chemical aspects of oil absorption of carbon sorbents on the water surface. Chemistry and Technology of Water 24: 22-31.

7. Tsai WT, Chang CY, Lee SL (1997) Preparation and Characterization of Activated Carbons from Corn Cob. Ibid 35: 1198-1200. 